\title{
Actinomycosis complicating chronic diverticulitis of the sigmoid colon: a missed association?
}

\author{
I. Samuel, ${ }^{1}$ M.F. Dixon ${ }^{2}$ and E.A. Benson ${ }^{1}$ \\ ${ }^{1}$ Department of Surgery, Leeds General Infirmary and ${ }^{2}$ Department of Pathology, University of Leeds, \\ Leeds, $U K$
}

Summary: The diagnosis and treatment of abdominal actinomycosis is difficult. We report a case of chronic diverticulitis of the sigmoid colon with stricture formation and obstruction, which was complicated by intramural actinomycosis. We suggest that diverticular strictures and actinomycosis may coexist more often than the literature suggests.

\section{Introduction}

Sigmoid diverticulitis is a common condition. Abdominal actinomycosis, though rare, poses problems of diagnosis and treatment, and has a high morbidity. ${ }^{1,2}$ The development of actinomycosis following perforated acute diverticulitis is well documented. ${ }^{2-4}$ We report a case of actinomycosis complicating chronic diverticulitis. Only one similar case has to our knowledge been previously reported.

\section{Case report}

A female aged 63 years was admitted with colicky lower abdominal pain, altered bowel habit, and a palpable mass per rectum. Sigmoidoscopy was obscured by faeces. Abdominal radiographs suggested large bowel obstruction, and a barium enema demonstrated complete sigmoid obstruction. The most likely diagnosis was considered to be carcinoma of the sigmoid colon.

At laparotomy a sigmoid mass was found with surrounding adherence, mesosigmoid induration and lymph node enlargement. An anterior resection, with a transverse loop colostomy, was performed. Post-operatively a gastrografin enema showed a small anastomotic leak which healed spontaneously.

Histology showed no evidence of neoplasia.

Correspondence: I. Samuel, F.R.C.S., Surgical Firm I, Directorate of Surgery, St Thomas' Hospital, London SE1 7EH, UK

Accepted: 1 July 1991
There was a $12 \mathrm{~cm}$ annular stricture with marked muscular hypertrophy. Numerous diverticula were present, some of which were inflamed, contained pus, and were surrounded by fibrosis. Actinomycotic colonies were seen in the bowel wall (Figure 1).

Intravenous penicillin was given, followed by oral ampicillin for 3 months. After checking the anastomosis with a gastrografin enema, the colostomy was closed. Subsequently, an anastomotic stricture developed, biopsies from which showed non-specific chronic inflammation. Two years later she remains asymptomatic.

\section{Discussion}

Actinomycosis in man is caused by Actinomyces israelii, a saprophyte of the mouth and gastrointestinal tract and an opportunistic pathogen. ${ }^{1}$ Abdominal actinomycosis is usually preceded by perforation or surgery of the gastrointestinal tract. $^{2,3}$

We report a patient with chronic diverticulitis of the sigmoid colon who presented with a mass, stricture and obstruction, in whom histology of the resected specimen showed actinomycosis within the bowel wall. Only one similar case has been previously reported..$^{5}$ The clinical presentation is different from that of actinomycosis following perforated acute diverticulitis, which is characterized by the development of fistulae or paracolic abscesses weeks or months after the acute episode. $^{2-4}$

The actinomycosis in our patient may have contributed to the development of the complications of fibrosis and stricture formation, obstruc- 
tion, anastomotic leakage and post-operative stricturing. As biopsies of actinomycotic tissue are frequently negative, ${ }^{2,4}$ and resection specimens from cases of diverticulitis are not widely sampled for histological examination, it is possible that chronic diverticulitis is complicated by actinomycosis more frequently than is thought.
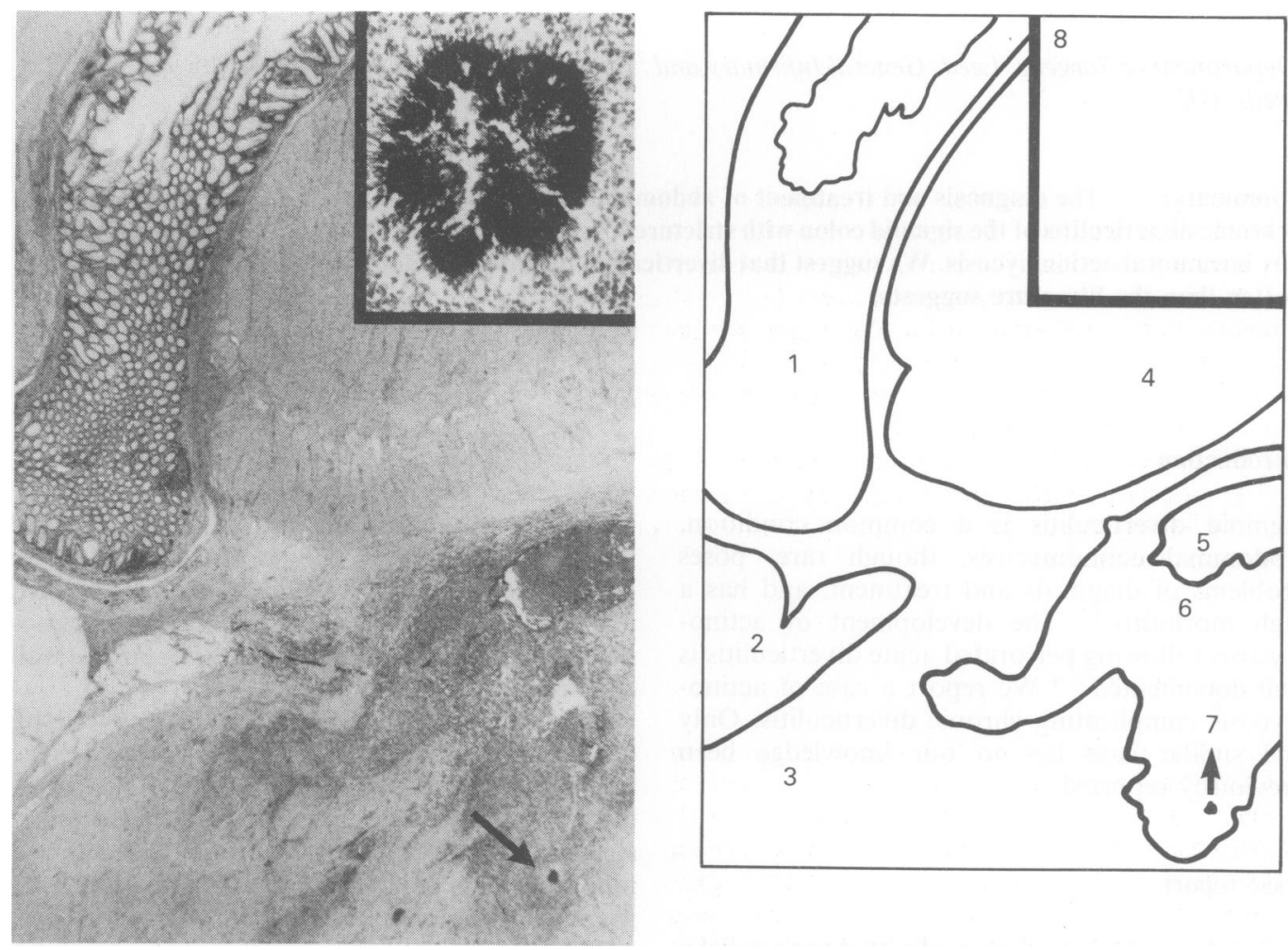

Figure 1 Low power view of a representative intact diverticulum penetrating through the muscularis propria. Outside the muscle coat there is suppuration and fibrosis surrounding 'colonies' of actinomycosis (arrowed) $(\mathrm{H} \& \mathrm{E} \times 4)$.

The inset shows a Grocott stained colony showing the radiating hyphae-like bacterial filaments $(x 64)$. The line diagram indicates: 1 . diverticulum (tangentially cut). 2. adipose tissue. 3. fibrous tissue. 4 . hypertrophied smooth muscle of the muscularis propria. 5. pus. 6. granulation tissue and inflammatory cells. 7. actinomycotic colony and 8 . inset.

\section{References}

1. Stringer, M.D. \& Cameron, A.E.P. Abdominal actinomycosis: a forgotten disease? Br J Hosp Med 1987, 38: 125-127.

2. Brown, J.R. Human actinomycosis: a study of 181 subjects. Hum Pathol 1973, 4: 319-330.

3. Gingold, B.S. \& Fazio, V.W. Abdominal actinomycosis: a complication of colonic perforation. Dis Colon Rectum 1978, 21: $374-376$.
4. Putman, H.C., Dockerty, M.B. \& Waugh, J.M. Abdominal actinomycosis: an analysis of 122 cases. Surgery 1950, 28: $781-800$.

5. Powers, P.W., Kramer, S.G. \& Drake, W.L. Actinomycosis of the sigmoid colon. Am J Surg 1961, 102: 713-715. 